\title{
Whole-genome sequencing for prediction of Mycobacterium tuberculosis drug susceptibility and resistance: a retrospective cohort study
}

\begin{abstract}
Timothy M Walker*, Thomas A Kohl*, Shaheed V Omar*, Jessica Hedge*, Carlos Del Ojo Elias, Phelim Bradley, Zamin Iqbal, Silke Feverriegel, Katherine E Niehaus, Daniel J Wilson, David A Clifton, Georgia Kapatai, Camilla L C Ip, Rory Bowden, Francis A Drobniewski, Caroline Allix-Béguec, Cyril Gaudin, Julian Parkhill, Roland Diel, Philip Supply, Derrick W Crook, E Grace Smith, A Sarah Walker, Nazir Ismailt, Stefan Niemannt, Tim E A Petot, and the Modernizing Medical Microbiology (MMM) Informatics Groupł
\end{abstract}

\section{Summary}

Background Diagnosing drug-resistance remains an obstacle to the elimination of tuberculosis. Phenotypic drugsusceptibility testing is slow and expensive, and commercial genotypic assays screen only common resistancedetermining mutations. We used whole-genome sequencing to characterise common and rare mutations predicting drug resistance, or consistency with susceptibility, for all first-line and second-line drugs for tuberculosis.

Methods Between Sept 1, 2010, and Dec 1, 2013, we sequenced a training set of 2099 Mycobacterium tuberculosis genomes. For 23 candidate genes identified from the drug-resistance scientific literature, we algorithmically characterised genetic mutations as not conferring resistance (benign), resistance determinants, or uncharacterised. We then assessed the ability of these characterisations to predict phenotypic drug-susceptibility testing for an independent validation set of 1552 genomes. We sought mutations under similar selection pressure to those characterised as resistance determinants outside candidate genes to account for residual phenotypic resistance.

Findings We characterised 120 training-set mutations as resistance determining, and 772 as benign. With these mutations, we could predict $89.2 \%$ of the validation-set phenotypes with a mean $\mathbf{9 2 . 3 \%}$ sensitivity (95\% CI $\mathbf{9 0 \cdot 7 - 9 3 \cdot 7 )}$ and $98 \cdot 4 \%$ specificity $(98 \cdot 1-98 \cdot 7) \cdot 10 \cdot 8 \%$ of validation-set phenotypes could not be predicted because uncharacterised mutations were present. With an in-silico comparison, characterised resistance determinants had higher sensitivity than the mutations from three line-probe assays (85.1\% vs $81 \cdot 6 \%$ ). No additional resistance determinants were identified among mutations under selection pressure in non-candidate genes.

Interpretation A broad catalogue of genetic mutations enable data from whole-genome sequencing to be used clinically to predict drug resistance, drug susceptibility, or to identify drug phenotypes that cannot yet be genetically predicted. This approach could be integrated into routine diagnostic workflows, phasing out phenotypic drugsusceptibility testing while reporting drug resistance early.

Funding Wellcome Trust, National Institute of Health Research, Medical Research Council, and the European Union.

Copyright (C) Walker et al. Open Access article distributed under the terms of CC-BY.

\section{Introduction}

WHO's target is to end the tuberculosis epidemic by 2035 . Multidrug-resistant tuberculosis poses the greatest obstacle to success, with an estimated 480000 cases worldwide in 2013 alone. ${ }^{1}$ Phenotypic drug-susceptibility testing for Mycobacterium tuberculosis can take many weeks, and access to the necessary laboratory facilities in countries with the greatest disease burden is often scarce. ${ }^{1}$ Although genotypic assays are faster and have diagnostic usefulness in both high-income and low-income countries, ${ }^{2-4}$ these assays screen a small number of genetic loci commonly associated with drug resistance, but are not designed to identify or exclude resistance by other mechanisms. ${ }^{5,6}$ Culture-based drug-susceptibility testing thus remains the gold-standard assay for testing resistance.

Whole-genome sequencing enables the screening of known resistance-associated loci while also providing opportunities to characterise other loci as predictive of resistance or not. ${ }^{2,7,8}$ To assess whether data from wholegenome sequencing can be used clinically to predict both drug resistance and drug susceptibility, we characterised the genetic variation in a large training set of samples and validated the findings by predicting phenotypes in an independent dataset.

\section{Methods}

Sample selection and processing sequences from the UK, Sierra Leone, South Africa Germany, and Uzbekistan, representing all seven global clades (appendix 1). ${ }^{9}$ We did phenotypic drug-susceptibility testing at reference laboratories in each of the countries (appendix 1) using the WHO-endorsed proportion method in an automated Mycobacterial Growth Indicator
We included $3651 M$ tuberculosis complex genome

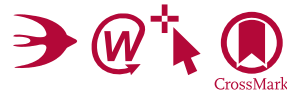

oa

Lancet Infect Dis 2015;

15: 1193-1202

Published Online

June 24, 2015

http://dx.doi.org/10.1016/

S1473-3099(15)00062-6

See Comment page 1124

${ }^{*}$ Contributed equally

†Contributed equally

¥For members see end of paper

Nuffield Department of

Medicine, University of Oxford, John Radcliffe Hospital, Oxford, UK (T M Walker MRCP,

J Hedge PhD, C Del Ojo Elias MSc, D) Wilson DPhil,

D W Crook FRCPath,

A S Walker PhD, T E A Peto FRCP); Molecular Mycobacteriology,

Forschungszentrum Borstel,

Leibniz-Zentrum für Medizin

und Biowissenschaften,

Borstel, Germany (T A Kohl PhD, S Feuerriegel PhD,

S Niemann PhD); Centre for Tuberculosis, National Institute for Communicable Diseases, Johannesburg, South Africa (SV Omar MSc, N Ismail FCPath); Wellcome Trust Centre for Human Genetics, University of Oxford, Oxford, UK (P Bradley MPhil, Z lqbal DPhil, ( L C Ip PhD, R Bowden PhD); German Center for Infection Research, Borstel Site, Borstel, Germany (S Feuerriegel, S Niemann); Institute of Biomedical Engineering, Department of Engineering Science, University of Oxford, Oxford, UK (KE Niehaus MS, D A Clifton DPhil); Microbiology Services, Public Health England, London, UK (G Kapatai PhD); Public Health England National Mycobacterial Reference Laboratory, Queen Mary's School of Medicine and Dentistry, London, UK (F A Drobniewski PhD); Department of Infectious Diseases, Imperial College, 
London, UK (F A Drobniewski); Genoscreen, Lille, France (C Allix-Béguec PhD, ( Gaudin PhD, P Supply PhD); Wellcome Trust Sanger Institute, Hinxton, UK (J Parkhill PhD); Institute for Epidemiology, University Medical Hospital SchleswigHolstein, Airway Research Center North, Kiel, Germany (R Diel PhD); Centre National de la Recherche Scientifique, Lille, France (P Supply); INSERM, Université de Lille, and Campus de l'Institut Pasteur de Lille, Center for Infection and Immunity of Lille, Lille, France (P Supply); National Institute of Health Research Oxford Biomedical Research Centre, John Radcliffe Hospital, Oxford, UK (D W Crook, A S Walker, T E A Peto); Public Health England West Midlands Public Health Laboratory, Heartlands Hospital, Birmingham, UK (EG Smith FRCPath); and Department of Medical Microbiology, University of Pretoria, Pretoria, South Africa (N Ismail) Correspondence to:

Dr Timothy M Walker, Nuffield Department of Medicine, University of Oxford, John Radcliffe Hospital, Oxford OX3 9DU, UK timothy.walker@ndm.ox.ac.uk

For the Tuberculosis Drug Resistance Mutation Database see https://tbdreamdb.ki.se

See Online for appendix 1
Research in context

\section{Evidence before this study}

We searched the PubMed database for studies published before April, 2015, using the terms "whole genome sequencing", "tuberculosis", "drug resistance", "drug susceptibility", "prediction", and "discovery". Much of the scientific literature on drug resistance in tuberculosis up to 2010 has concentrated on identifying resistance-conferring mutations, and has been summarised in the Tuberculosis Drug Resistance Mutation Database. This database includes the small number of common drug-resistance mutations upon which the design of commercial molecular assays such as the Hain MTBDRplus line-probe (Nehren, Germany) and the Cepheid MTB/RIF GeneXpert (Sunnyvale, CA, USA) are based. As these assays only screen for phenotypic resistance, and not phenotypic susceptibility, expensive and slow phenotypic drug-susceptibility testing remains necessary to define which drugs will effectively treat patients. Some studies have used DNA sequencing techniques to predict phenotypic resistance from a wider set of known genotypic-resistance mechanisms, and to discover new drugresistance mechanisms. One recent study made an important contribution by seeking to characterise each mutation in the pncA gene, relevant to the key first-line drug pyrazinamide, as either conferring resistance or not conferring resistance, thereby raising the prospect of predicting both drug resistance and drug susceptibility from genetic data, and reducing the need for phenotyping for pyrazinamide.

\section{Added value of this study}

Our study expands on these findings by examining mutations for all first-line and second-line antituberculosis drugs. By using a large number of whole-genome sequences we were able to control for population structure and characterise mutations within relevant genes identified in the scientific literature as either conferring resistance or consistent with drug susceptibility. We are also able to search the rest of the genome for additional genes of relevance to drug resistance. Through characterisation of all mutations, this approach can establish which drugs will be effective against clinical isolates and, because it is iteratively updatable, can result in fewer isolates needing phenotypic drug-susceptibility testing.

\section{Implications of all the available evidence}

The growing body of knowledge on mutations conferring drug resistance or consistent with susceptibility will provide the basis from which a near-definitive genotypic assay can be designed that will eventually bypass the need for phenotypic drug-susceptibility testing. Early results from this study have provided information for a pilot of drug-susceptibility testing based on whole-genome sequencing in the UK, and, as portable whole-genome sequencing platforms become available, could transform drug-susceptibility testing in low-income settings where many of the world's patients with tuberculosis live, and where many still rely on empirical treatment regimens.
Tube 960 system (Becton Dickinson), on solid LowensteinJensen media, or the resistance ratio method. UK samples were tested for routine patient-care purposes, and nonUK samples for research. Drug-susceptibility testing for one or more of isoniazid, rifampicin, ethambutol, pyrazinamide, streptomycin, ciprofloxacin, moxifloxacin, ofloxacin, amikacin, capreomycin, and kanamycin was available for each isolate. We prepared DNA for sequencing using the Nuclisens EasyMag (Biomerieux, France) following the manufacturer's protocol, the Fuji Quickgene kit (Kurabo Biomedical, Osaka, Japan), or the cetyltrimethylammonium bromide method of DNA purification (as previously described). ${ }^{10,11}$ We used Illumina (San Diego, CA, USA) sequencing platforms at the Wellcome Trust Centre for Human Genetics (Oxford, UK), the Wellcome Trust Sanger Institute (Hinxton, UK), the Forschungszentrum Borstel (Borstel, Germany); Genoscreen (Lille, France), and the National Institute for Communicable Diseases (Johannesburg, South Africa).

Paired-end reads were mapped with Stampy ${ }^{12}$ (version 1.0.17) to the H37Rv (GenBank NC000962.2) reference genome, which was phenotypically susceptible to all drugs of interest. Repetitive genome sections were defined by self-self BLAST and masked. We excluded isolates with less than $88 \%$ mapped coverage of the reference genome (appendix 1). Base calls were made with SAMtools mpileup ${ }^{13}$ (version 0.1 .18 ), requiring a minimum-read depth of $5 \times$, including at least one read on each strand. Where an alternative base represented more than $10 \%$ of read depth, mixed base calls were made. These base calls were only included in the downstream analysis if, in at least one other isolate, they constituted more than $90 \%$ of read depth (ie, a nonmixed base call). We identified insertions and deletions with Cortex..$^{14}$ One inconsistent base call was identified across 202 technical replicates (error $<1 \times 10^{-9}$ per base) We used RAxML (version 8.0.5) to reconstruct the phylogeny under a general time reversible model with rate variation modelled by fixed-rate categories. ${ }^{15}$ We estimated the frequency of each single nucleotide polymorphism arising in the phylogeny (ie, homoplasy) using maximum-likelihood ancestral site reconstruction. ${ }^{16}$ No ethics approval was required for this study.

\section{Characterisation of mutations}

Identification of resistance-causing single-nucleotide polymorphisms in clonal bacteria using genome-wide association studies is challenging. ${ }^{17}$ We therefore first focused on 23 candidate genes and their promoter regions (figure 1), each with at least one previously described drug-resistance mutation (appendix 1). We devised an algorithm to characterise all mutations for these genes compared with the pan-susceptible reference genome at the level of single-nucleotide polymorphisms 
in promoter regions, aminoacids in genes, or insertions and deletions. We characterised mutations separately for each relevant drug without taking previous findings about specific mutations from the scientific literature into account, and then used these results to predict drugsusceptibility test results in other independent samples.

Samples were obtained in batches over time. We first used a training set of 2099 samples from the UK, Sierra Leone, and South Africa to characterise mutations as resistance determining or not. We then obtained a validation set of 1552 samples from Germany, Uzbekistan, and South Africa, against which characterisations were assessed. To check for bias resulting from the composition of sets, we repeated analyses after switching training and validation sets, and then repeated a further 100 times, randomly allocating samples to equally sized training and validation sets (appendix 1).

To algorithmically characterise mutations in the training set, we first assumed that synonymous and lineagedefining mutations do not cause resistance, unless the lineage-defining mutations were associated with lineagespecific resistance (eg, pyrazinamide in Mycobacterium bovis, ${ }^{18}$ appendix 1). After these mutations were labelled as benign and set aside, we assessed the remaining mutations within each group of genes relevant to each drug in turn, and generated hypotheses regarding associations with drug resistance (to be tested in the validation set). A mutation was characterised as resistance determining if it occurred as the only mutation across all relevant candidate genes in at least one phenotypically resistant isolate in the training set. Mixed-base calls were regarded as mutations rather than wild types. Since mutations that do not cause resistance can clearly co-occur with those that do, mutations were characterised as benign if they take place only in phenotypically susceptible isolates, or where all isolates were phenotypically susceptible when a mutation occurred alone. These benign mutations were then also set aside and the analysis repeated to potentially reveal further resistancedetermining mutations (appendix 1). Where resistance could not be accounted for by a characterised resistance determinant, evidence of synergy between mutations, or of co-occurring compensatory mutations, was sought by manual inspection of sequences.

Validation-set isolates containing mutations characterised in the training set as resistance determining were predicted resistant, and those containing no mutations, or only mutations characterised as benign, were predicted susceptible. Isolates containing uncharacterised mutations were hence not predicted unless co-occurring with resistance determinants. We then made a comparison with predictions based only on mutations probed by the Genotype MTBDRplus, MTBDRsl (HAIN Lifesciences, Germany), and AID (AID Diagnostika, Germany) line-probe assays. Finally, all 3651 isolates were combined and the algorithm reapplied.

Because some resistant phenotypes might not be attributable to mutations in the 23 genes, the remaining genome was explored for potential explanatory mutations. Because resistance-causing mutations are likely to be under positive selection pressure, these mutations are also the most likely to arise repeatedly, independently in the phylogeny. ${ }^{8}$ Focusing our search for additional resistance determinants on these homoplasic mutations, we quantified the frequency of homoplasic events for each mutation in the genome and compared the frequency recorded across the 23 genes and among characterised resistance determinants to that among mutations in genes or open reading frames and functional RNA molecules elsewhere in the genome. ${ }^{19}$ Analyses were done with Stata 13.1 (StataCorp, Texas).

\section{Role of the funding source}

The funder of the study had no role in study design, data collection, data analysis, data interpretation, or writing of the report. The corresponding author had full access to all the data in the study and had final responsibility for the decision to submit for publication.

\section{Results}

$2099 M$ tuberculosis isolates were sequenced as a training set, within which 1414 independent strains could be identified by clustering isolates within five single-nucleotide

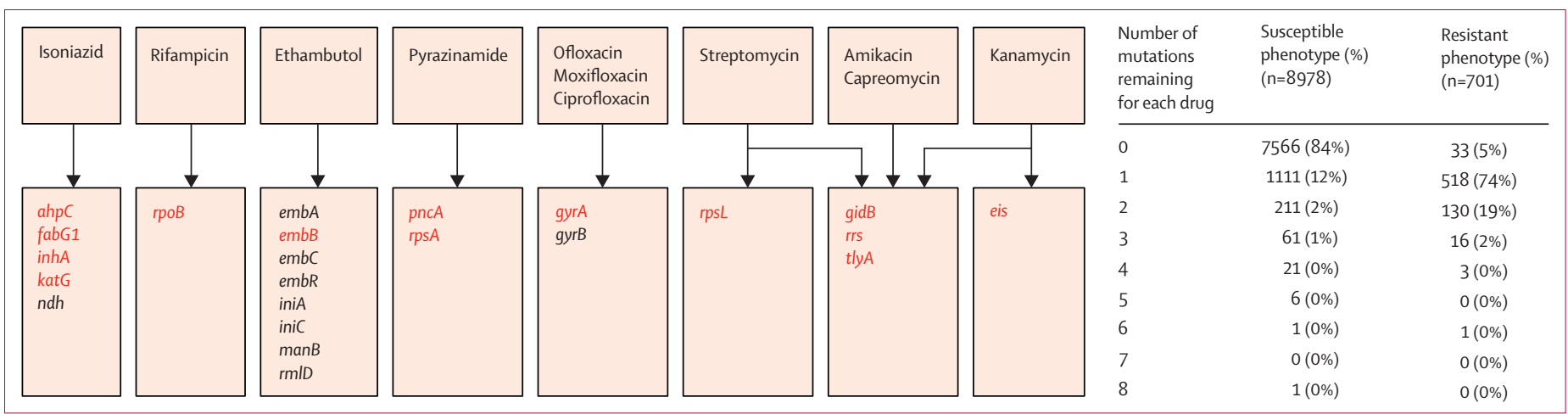

Figure 1: Candidate genes and mutations

The number of potentially predictive mutations in genes relevant to each drug after lineage-defining and synonymous mutations have been set aside and are shown by susceptible and resistant phenotypes for 2099 training-set isolates. Genes from which one or more of the 120 resistance-determining mutations were algorithmically characterised are coloured red. 
polymorphisms of another. ${ }^{11} 382$ (18.2\%) were phenotypically resistant to at least one drug, 91 (4.3\%) were multidrug resistant, and four $(0 \cdot 2 \%)$ were extensively drug resistant, making a total of 701 (7.2\%) resistant phenotypes. 8978 susceptible phenotypes were identified.

The individual steps in the mutation identification algorithm are detailed in appendix 1 . After all lineagedefining and synonymous mutations were set aside, apart from pncA H57D and rpsA A440T because these were present in all $M$ bovis isolates (intrinsically pyrazinamide resistant), 991 mutations (counting mutations more than once if relevant to more than one drug) were left for consideration. One mutation remained in 518 (74\%) of resistant and 1111 (12\%) of susceptible phenotypes, whereas no mutations remained for 7566 (84\%) of susceptible and 33 (5\%) of resistant phenotypes (figure 1). 112 mutations were thereby classified as resistance determining, and 772 were classified as benign. After setting these benign mutations aside, six additional mutations were classified as resistance determining, but no further mutations could be characterised by repeating the algorithm again. 101 mutations thus remained unclassified, of which 60 co-occurred only with resistance determinants, compatible with a possible compensatory role (appendix 1). The 120 resistance-determining mutations (including $p n c$ A H57D and rpsA A440T) were spread across just 14 candidate genes (figure 1), with $79(66 \%)$ of 120 having previously been described as resistance determining in the scientific literature (appendix 1).
At least one resistance determinant was present in $658(93.9 \%)$ of 701 resistant training-set phenotypes. $33(4.7 \%)$ of 701 resistant phenotypes remained unaccounted for with no relevant mutations in relevant genes, and $10(1.4 \%)$ of 701 could not be algorithmically unravelled because they contained more than one relevant mutation. Six of these contained mutations associated with resistance in the scientific literature (appendix 1).

We also noted resistance-determining mutations in 121 susceptible phenotypes. Such phenotypic variability was most evident for isolates containing $e m b B$ M306I and $r p o B$ I491F. 34 (68\%) of 50 containing embB M306I were phenotypically susceptible to ethambutol and 19 (83\%) of 23 containing rpoB I491F were phenotypically susceptible to rifampicin (appendix 1). Although mutations elsewhere in the genome might account for such variability through epistasis, a subset of eight ethambutol-resistant and three rifampicin-resistant isolates each had a genetically indistinguishable (ie, no single-nucleotide polymorphisms) but phenotypically susceptible paired isolate. Such phenotypic changes without genotypic changes suggest poor phenotypic reproducibility for these mutations at least. ${ }^{20}$

To assess their accuracy, we used training-set characterisations to predict phenotypes for an independent validation set of 1552 isolates that included 449 isolates that were phenotypically resistant to at least one drug, 284 that were multidrug resistant and three that were extensively drug resistant (table, figure 2, appendix 1). $58(48 \cdot 3 \%)$ of 120 mutations characterised as resistance determining in the training set, and $175(22 \cdot 7 \%)$ of

\begin{tabular}{|c|c|c|c|c|c|c|c|c|c|c|c|c|c|c|c|c|c|}
\hline & \multicolumn{6}{|c|}{ Phenotypically resistant } & \multicolumn{6}{|c|}{ Phenotypically sensitive } & \multicolumn{2}{|l|}{ All } & \multicolumn{2}{|c|}{ Excluding uncharacterised } & \multirow[t]{3}{*}{ Uncharacterised } \\
\hline & \multicolumn{5}{|c|}{ Genotype } & \multirow[t]{2}{*}{ Total } & \multicolumn{5}{|c|}{ Genotype } & \multirow[t]{2}{*}{ Total } & \multirow[t]{2}{*}{ Sensitivity } & \multirow[t]{2}{*}{ Specificity } & \multirow[t]{2}{*}{ Sensitivity } & \multirow[t]{2}{*}{ Specificity } & \\
\hline & $\mathrm{R}$ & $\mathrm{R}_{\mathrm{x}}$ & $\mathrm{S}_{0}$ & $\mathrm{~S}_{\mathrm{B}}$ & U & & $\mathrm{R}$ & $\mathrm{R}_{\mathrm{x}}$ & $\mathrm{S}_{0}$ & $\mathrm{~S}_{\mathrm{B}}$ & U & & & & & & \\
\hline Isoniazid & 305 & 5 & 18 & 1 & 35 & 364 & 19 & 0 & 1065 & 52 & 52 & 1188 & $\begin{array}{l}85 \cdot 2 \\
(81 \cdot 1-88 \cdot 7)\end{array}$ & $\begin{array}{c}98 \cdot 4 \\
(97 \cdot 5-99 \cdot 0)\end{array}$ & $\begin{array}{l}94 \cdot 2 \\
(91 \cdot 1-96 \cdot 5)\end{array}$ & $\begin{array}{l}98 \cdot 3 \\
(97 \cdot 4-99 \cdot 0)\end{array}$ & $5 \cdot 6 \%$ \\
\hline Rifampicin & 263 & 12 & 8 & 1 & 16 & 300 & 9 & 1 & 1200 & 4 & 38 & 1252 & $\begin{array}{l}91 \cdot 7 \\
(87 \cdot 9-94 \cdot 5)\end{array}$ & $\begin{array}{l}99 \cdot 2 \\
(98 \cdot 5-99 \cdot 6)\end{array}$ & $\begin{array}{l}96 \cdot 8 \\
(94 \cdot 1-98 \cdot 5)\end{array}$ & $\begin{array}{l}99 \cdot 2 \\
(98 \cdot 5-99 \cdot 6)\end{array}$ & $3.5 \%$ \\
\hline Ethambutol & 152 & 6 & 7 & 1 & 26 & 192 & 62 & 5 & 1003 & 79 & 210 & 1359 & $\begin{array}{l}82 \cdot 3 \\
(76 \cdot 1-87 \cdot 4)\end{array}$ & $\begin{array}{c}95 \cdot 1 \\
(93 \cdot 8-96 \cdot 2)\end{array}$ & $\begin{array}{l}95 \cdot 2 \\
(90 \cdot 7-97 \cdot 9)\end{array}$ & $\begin{array}{l}94 \cdot 2 \\
(92 \cdot 7-95 \cdot 4)\end{array}$ & $15 \cdot 2 \%$ \\
\hline Pyrazinamide & 31 & 12 & 27 & 5 & 104 & 179 & 2 & 0 & 1218 & 67 & 83 & 1370 & $\begin{array}{l}24 \cdot 0 \\
(17 \cdot 9-30 \cdot 9)\end{array}$ & $\begin{array}{c}99 \cdot 9 \\
(95 \cdot 5-100 \cdot 0)\end{array}$ & $\begin{array}{l}57 \cdot 3 \\
(45 \cdot 3-68 \cdot 7)\end{array}$ & $\begin{array}{c}99 \cdot 8 \\
(99 \cdot 4-100 \cdot 0)\end{array}$ & $12 \cdot 1 \%$ \\
\hline Streptomycin & 278 & 6 & 6 & 9 & 49 & 348 & 10 & 1 & 970 & 34 & 189 & 1204 & $\begin{array}{l}81 \cdot 6 \\
(77 \cdot 1-85 \cdot 5)\end{array}$ & $\begin{array}{c}99 \cdot 1 \\
(98 \cdot 4-99 \cdot 5)\end{array}$ & $\begin{array}{l}95 \cdot 0 \\
(91 \cdot 9-97 \cdot 2)\end{array}$ & $\begin{array}{c}98 \cdot 9 \\
(98 \cdot 1-99 \cdot 4)\end{array}$ & $15 \cdot 3 \%$ \\
\hline Ofloxacin & 2 & 3 & 4 & 2 & 0 & 11 & 0 & 0 & 489 & 134 & 38 & 661 & $\begin{array}{l}45 \cdot 5 \\
(16 \cdot 7-76 \cdot 6)\end{array}$ & $\begin{array}{l}100 \cdot 0 \\
(99 \cdot 4-100 \cdot 0)\end{array}$ & $\begin{array}{l}45 \cdot 5 \\
(16 \cdot 7-76 \cdot 6)\end{array}$ & $\begin{array}{l}100 \cdot 0 \\
(99 \cdot 4-100 \cdot 0)\end{array}$ & $5.7 \%$ \\
\hline Amikacin & 36 & 16 & 5 & 0 & 2 & 59 & 1 & 2 & 427 & 38 & 140 & 608 & $\begin{array}{l}88 \cdot 1 \\
(77 \cdot 1-95 \cdot 1)\end{array}$ & $\begin{array}{c}99.5 \\
(98.6-99.9)\end{array}$ & $\begin{array}{l}91 \cdot 2 \\
(80 \cdot 7-97 \cdot 1)\end{array}$ & $\begin{array}{c}99 \cdot 4 \\
(98 \cdot 1-99 \cdot 9)\end{array}$ & $21 \cdot 3 \%$ \\
\hline Total & 1067 & 60 & 75 & 19 & 232 & 1453 & 103 & 9 & 6372 & 408 & 750 & 7642 & $\begin{array}{l}77 \cdot 6 \\
(75 \cdot 3-79 \cdot 7)\end{array}$ & $\begin{array}{l}98.5 \\
(98.2-98.8)\end{array}$ & $\begin{array}{l}92 \cdot 3 \\
(90 \cdot 7-93 \cdot 7)\end{array}$ & $\begin{array}{c}98 \cdot 4 \\
(98 \cdot 1-98 \cdot 7)\end{array}$ & $10 \cdot 8 \%$ \\
\hline
\end{tabular}

Total sensitivity and specificity data are weighted means ( $95 \% \mathrm{Cls}$ ). We investigated each drug separately by comparing the phenotype for each across isolates with this data available. The unit of analysis was therefore not an isolate, but a phenotype. $\mathrm{R}=$ resistance-determining mutation. $\mathrm{R}_{\mathrm{x}}=$ resistance determinant only as a mixed base call (heteroresistance). $\mathrm{S}_{0}=\mathrm{zero}$ mutations present. $\mathrm{S}_{\mathrm{z}}=$ only benign mutations present. $\mathrm{U}=$ uncharacterised mutations present in the absence of a resistance-determining mutation. Characterised mutations only exclude the $\mathrm{U}$ columns. To avoid double counting for several drugs from the same class, ofloxacin and amikacin were included as representatives of their antibiotic classes, because these had the most resistant phenotypes. Results for ciprofloxacin, moxifloxacin, kanamycin, and capreomycin are in the appendix. 
772 characterised as benign, recurred in validation-set isolates. These mutations predicted $89 \cdot 2 \%$ of validationset phenotypes as resistant or susceptible with a mean $92.3 \%$ sensitivity (95\% CI $90 \cdot 7-93 \cdot 7)$ and $98.4 \%$ specificity $(98 \cdot 1-98 \cdot 7)$, using ofloxacin and amikacin as representatives of their respective drug classes (table). The presence of uncharacterised mutations in validationset isolates prevented predictions for the remaining $10 \cdot 8 \%$ of phenotypes.

58 recurring resistance-determining mutations occurred across the resistant and susceptible validationset phenotypes (distribution listed in appendix 1). $54(93 \cdot 1 \%)$, including 12 not described in the scientific literature, accurately predicted at least one resistant validation-set phenotype. The proportion of phenotypes accurately predicted resistant varied substantially by drug (table). Predictions for pyrazinamide were the least sensitive. Of 34 pncA mutations characterised in the training set as resistance determinants, 12 recurred in the validation set. Although 43 (97.7\%) of 44 validationset isolates in which these mutations were noted were phenotypically resistant, these accounted for only $24 \%$ of pyrazinamide-resistant isolates in the validation set. Conversely, predictions for rifampicin were the most sensitive. $12(66 \cdot 7 \%)$ of 18 training-set characterised resistance determinants recurred in the validation set, successfully predicting phenotypic resistance in $275(96.5 \%)$ of 285 isolates in which these were noted, and accounting for $91.7 \%$ of rifampicin-resistant isolates in the validation set.

Of 1221 resistant phenotypes in the validation set, $94(7.7 \%)$ were incorrectly predicted susceptible. $20(21.3 \%)$ of 94 were due to mutations characterised as benign in the training set, and $74(78.7 \%)$ of 94 had no relevant mutations, suggesting either a phenotypic or labelling error, or a resistance mechanism outside candidate genes. Of 6892 susceptible validation-set phenotypes, $112(1.6 \%)$ were wrongly predicted resistant,

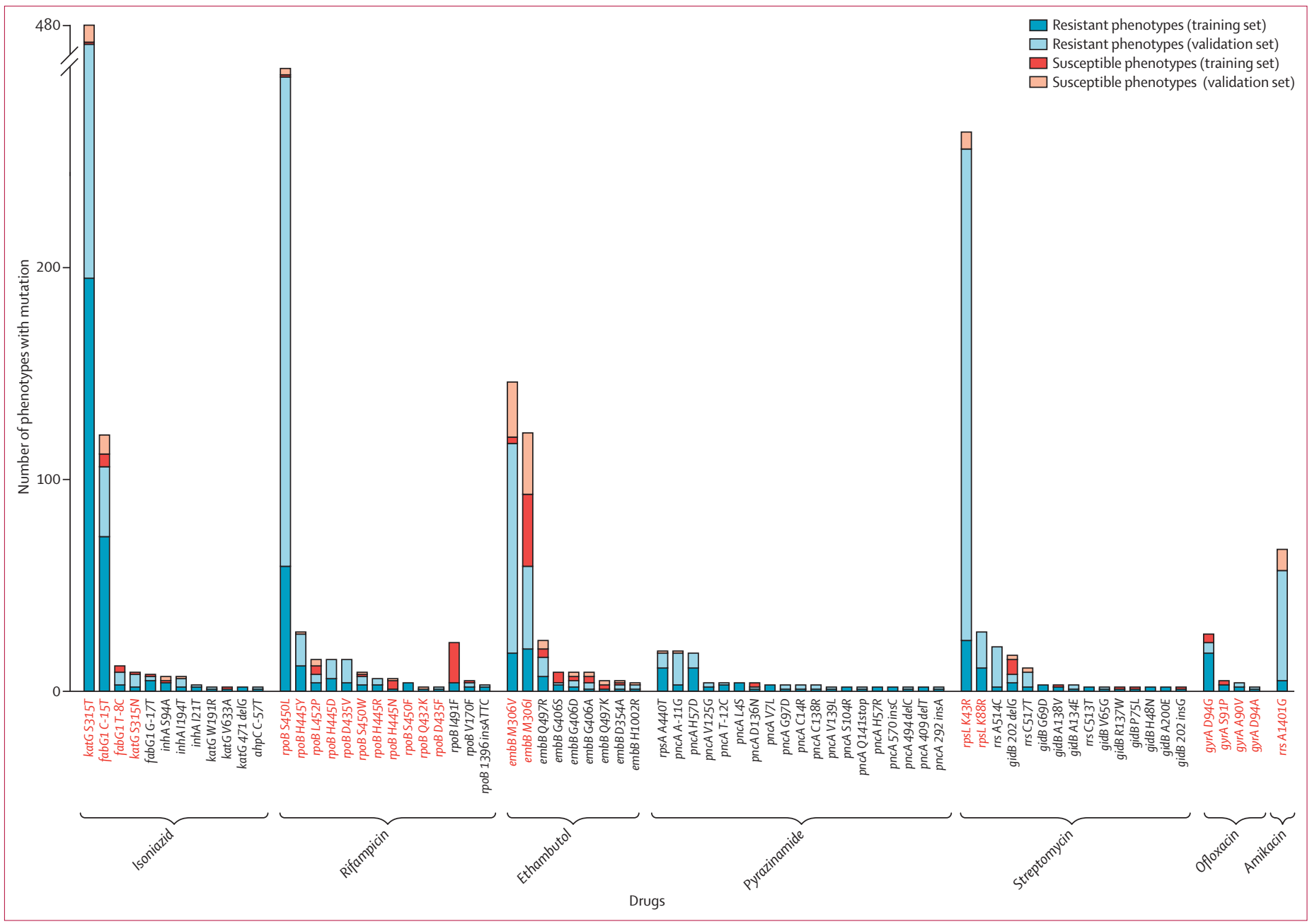

Figure 2: Resistance determinants in training and validation sets

Mutations probed by a line-probe assay are coloured red. Mutations that were only noted once in the training set and not again in the validation set (ie, with no additional information to validate them) are not shown. Of the quinolones and aminoglycosides, only ofloxacin and amikacin have been included as representatives of their class. 
$55(49.1 \%)$ of 112 contained mutations at embB M306, suggesting associated phenotypic variability. Eight (7.1\%), however, contained katG S315T, which is more likely to represent a labelling error rather than a phenotypic error (appendix 1). ${ }^{2}$ To assess whether these results depended on the allocation of isolates to training and validation sets, we repeated the algorithm using the validation set as the training set, and vice versa, predicting $93.1 \%$ of phenotypes with mean $92 \cdot 1 \%$ (95\% CI 90.1-93.7) sensitivity and $97 \cdot 9 \%(97 \cdot 6-98 \cdot 3)$ specificity. We did a further 100 iterations of the algorithm, on each occasion randomly allocating samples to training or validation sets. Over these 100 iterations, the mean proportion of predictable phenotypes in validation sets was $92 \cdot 7 \%$, and the mean sensitivity and specificity $92.4 \%$ (means are the same value as the medians; IQR 91.9-93.0) and $98 \cdot 2 \%$ (98.1-98·3), respectively (appendix 1 ).

In view of the consistency of these results, we compared the original predictions for the validation set with

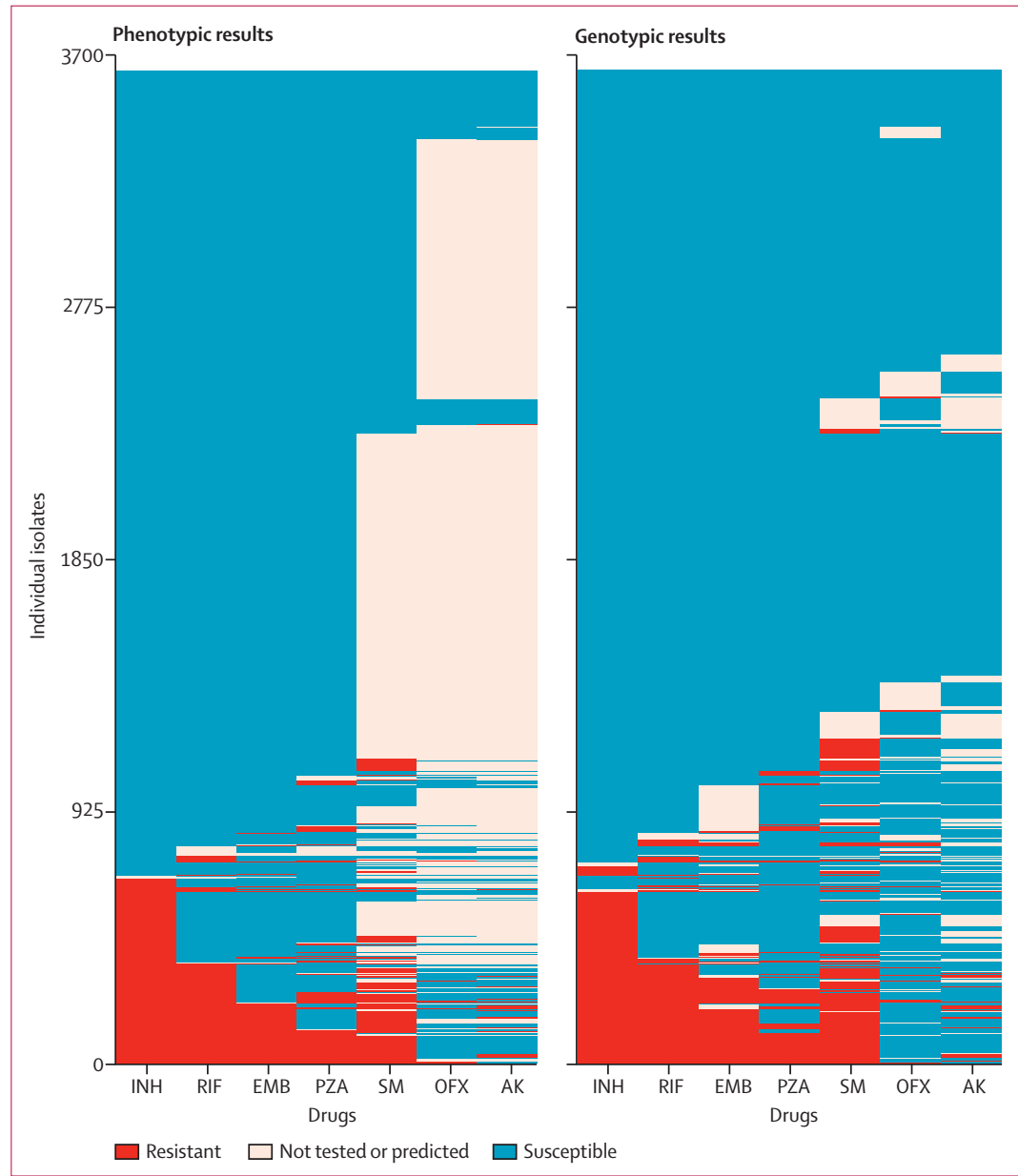

Figure 3: Phenotypic and genotypic antibiograms for all 3651 isolates

The left-hand panel shows the phenotypes for seven drugs for the 3651 isolates. The right-hand panel shows the genotypic predictions based on the mutations characterised after applying the algorithm to all 3651 isolates. $\mathrm{INH}=$ =isoniazid. $\mathrm{RIF}=$ rifampicin. $\mathrm{EMB}=$ =thambutol. $\mathrm{PZA}=$ pyrazinamide. $\mathrm{SM}=$ streptomycin. $\mathrm{OFX}=$ ofloxacin AK=amikacin. predictions based on mutations probed by three line-probe assays. With the exception of pyrazinamide, for which no line-probe assay exists, these assays collectively predicted validation-set phenotypes with mean $81.6 \%$ (95\% CI 79.4-83.7) sensitivity and $98 \cdot 0 \%(97 \cdot 6-98 \cdot 3)$ specificity, compared with $85 \cdot 1 \%(83 \cdot 0-87 \cdot 0)$ and $98 \cdot 2 \%(97 \cdot 9-98 \cdot 6)$ respectively, for the algorithmically characterised mutations based on whole-genome sequencing. However, unlike the line-probe assays, we could use the algorith mically identified mutations to unambiguously distinguish between benign and uncharacterised mutations, allowing further improvement to the results by restricting predictions to the $89.2 \%$ of predictable validation-set phenotypes. For these, the mean sensitivity and specificity, excluding pyrazinamide, were $94.6 \%(93 \cdot 1-95 \cdot 8)$ and $98 \cdot 0 \%(97 \cdot 6-98 \cdot 4)$, respectively (appendix 1$)$.

The algorithm was rerun for all 3651 isolates, which increased the number of mutations characterised as resistance determining from 120 to 232, and as benign from 772 to 1634. Among the resistance-determining mutations were three that had remained uncharacterised in the original training set, and 16 originally characterised as benign but recharacterised because of additional samples from the phenotypically resistant validation set containing only those mutations. Eight $(42 \cdot 1 \%)$ of these 19 mutations had been previously described as resistance determining in the scientific literature (appendix 1). Because all samples were included in this training set, no independent validation set remained, but predictions were made for the entire set itself. $96 \cdot 1 \%$ of phenotypes could be predicted with mean $94.8 \%(95 \%$ CI $93 \cdot 8-95 \cdot 7)$ sensitivity and $98 \cdot 0 \%$ specificity $(97 \cdot 7-98 \cdot 2$; figure 3 , appendix 1$)$.

We assessed all nucleotide positions across the phylogeny of all 3651 isolate samples for homoplasy to explore first whether characterised resistance determinants were under selection pressure, and then to identify which mutations beyond the 23 candidate genes might be similarly under selection pressure, and therefore plausibly resistance determining. Across the 23 concatenated candidate-gene sequences, $292(0 \cdot 8 \%)$ of 38257 nucleotide positions were homoplasic. These affected $63(52.5 \%)$ of 120 resistance determinants, $17(16 \cdot 8 \%)$ of 101 uncharacterised mutations, and $59(7.6 \%)$ of 772 benign mutations, as characterised in the training set (figure 4). Outside the 23 candidate genes, $5427(0 \cdot 1 \%)$ of 4373275 nucleotide positions were homoplasic, involving 2341 (59.3\%) of 3951 remaining genes in the genome.

To increase the probability of finding resistancedetermining mutations within this many genes, we identified the most homoplasic by summing the maximum number of homoplasic emergences affecting mutations across each gene. For ten of 14 genes providing the 120 resistance determinants in the training set there were a median 102 emergences (IQR 32-1070), placing them among the $34(1.4 \%)$ of 2364 most homoplasic genes. This compared with a median of five emergences (two to ten) 
for the other four of 14 genes, and four emergences (two to six) for the remaining genes in the genome.

We searched the 34 most homoplasic genes for nonsynonymous mutations that might account for any of the 33 unaccounted-for resistant phenotypes in the training set. These mutations were however predominantly associated with susceptible phenotypes in other isolates (median $12.5 \%$ phenotypically resistant, IQR 9-28\%) (appendix 1). Those most strongly associated with phenotypic resistance were rpoC G332R for ethambutol (five of six resistant), and phoR P186L (three of five) for isoniazid. However, all six isolates containing rpo C G332R were phenotypically resistant to rifampicin (all contained $r p o B$ S450L), suggesting that rpoC G332R was more likely to be a compensatory mutation for rifampicin, than the cause of ethambutol resistance. The two other isoniazidresistant isolates containing phoR P186L both also contained the derived resistance determinant fab G1 G-17T. No additional convincing resistance determinants were therefore noted.

\section{Discussion}

We used a training set of $2099 \mathrm{M}$ tuberculosis genomes to algorithmically characterise mutations across 23 candidate genes as either resistance determining or benign. These characterised mutations predicted $89.2 \%$ of phenotypes for an independent validation set of 1552 isolates with high sensitivity and specificity of $92 \cdot 3 \%(95 \%$ CI $90 \cdot 7-93 \cdot 7)$ and $98 \cdot 4 \%(98 \cdot 1-98 \cdot 7)$.

$84 \%$ of susceptible phenotypes contained no relevant mutations compared with the pan-susceptible reference, and $74 \%$ of resistant phenotypes contained exactly onethese findings were key to the characterisation of mutations. Phenotypes were successfully predicted because the same resistance determinants happened repeatedly, independently across isolates. Results were therefore largely independent of training and validationset composition.

The characterisation of all mutations offers advantages over line-probe assays and other commercial molecular assays. First, data from whole-genome sequencing can be screened for all resistance determinants, resulting in a higher sensitivity than for the mutations based on lineprobe assays alone. ${ }^{21,22}$ Second, although line-probe assays can suggest which drugs to avoid by screening a few key resistance-determining mutations, they leave some doubt about which drugs to give. By characterising mutations as benign, we can actively predict phenotypic susceptibility in some isolates, contrasting them from others containing uncharacterised mutations. Third, drug-susceptibility testing based on whole-genome sequencing can be done for additional and even novel drugs at no additional cost, contingent only on the knowledge base of characterised mutations. This wide application could be helpful when designing new treatment regimens. ${ }^{23,24}$

Despite the success of the algorithm, some mutations could not be correctly characterised, and some resistant

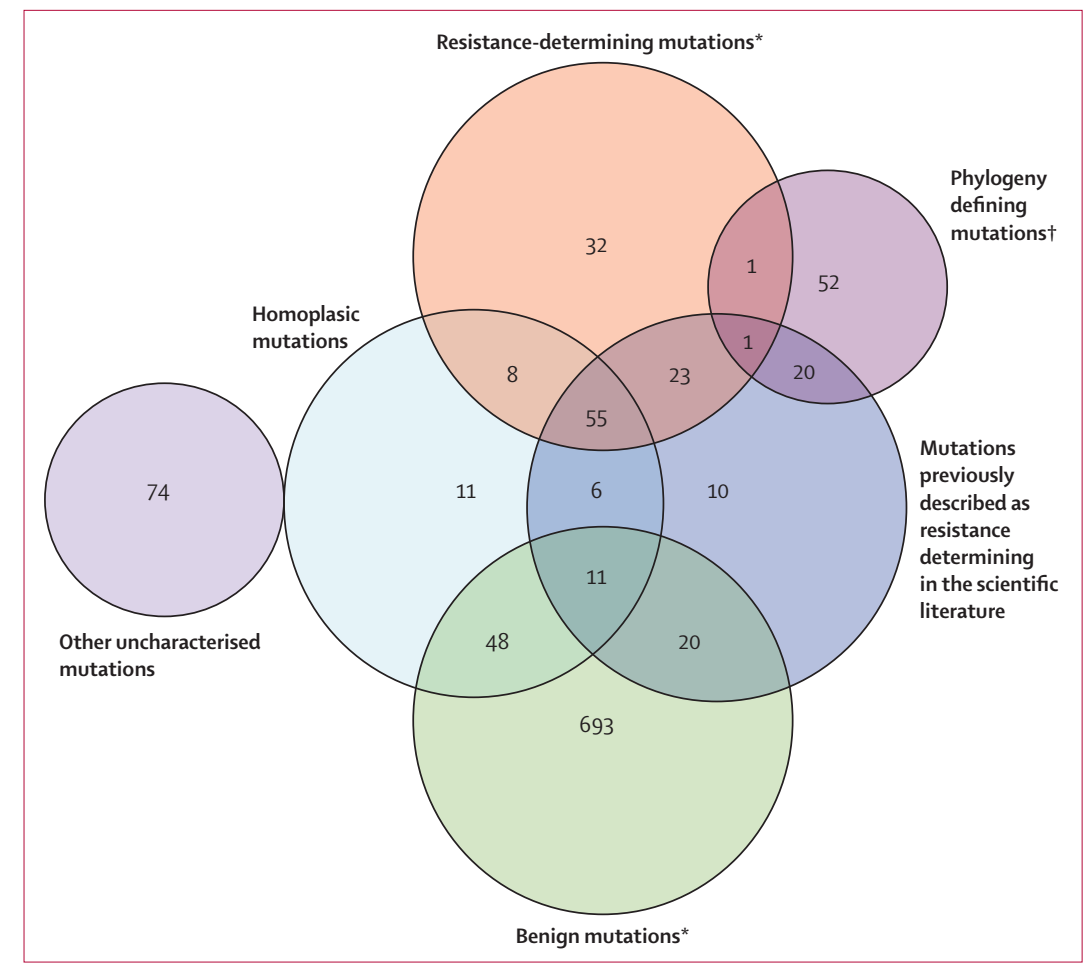

Figure 4: Training-set-characterised mutations

Numbers represent the number of mutations for each characterisation. *Among resistance determinants and benign mutations, 15 and 55 insertions and deletions, and 25 and 371 mutations seen in only one isolate respectively, were not or could not be assessed for homoplasy. $\operatorname{tgyrA}$ A384V defines the Indian Ocean lineage (all isolates in the lineage have this single-nucleotide polymorphism) but is also in one European American isolate rpsA A440T defines Mycobacterium bovis but is also in one Central Asian isolate. Both are thereby homoplasic.

phenotypes could not be ascribed a causative mutation. One reason is imperfect phenotypic drug-susceptibility testing, ${ }^{25,26}$ best shown by the weak association between $\mathrm{embB}$ M306I and ethambutol resistance noted both within and across study sites. ${ }^{20}$ Since the algorithm implicitly upweights single recordings of resistance over susceptibility, new samples could lead to the recharacterisation of mutations from benign to resistant, although rarely vice versa (appendix 1). Nevertheless, because whole-genome sequencing variant calling is highly reproducible, ${ }^{10,27}$ phenotypic variability around some mutations will become apparent in large datasets, including those with more resistance to second-line drugs, for which predictions could be recast within a Bayesian analysis framework. ${ }^{28}$ The reproducibility and robustness of sequencing data also has the potential for in-vitro phenotypic techniques to be recalibrated and reassessed. However, if further evidence for the effect of particular mutations is warranted, additional approaches such as in-vitro mutagenesis or crystallographic protein analysis might still be needed. ${ }^{8,29}$

Another possible reason is the presence of mechanisms of resistance outside candidate genes. The homoplasic signal of selection pressure has previously been used by Farhat and colleagues to associate genome-wide mutations with resistance. ${ }^{8}$ The ponA1 mutations they 
associated with rifampicin resistance were, however, not homoplasic in these data, and the only non-synonymous ponA1 mutation that was homoplasic (ponA1 D24N) was only present in five isolates susceptible to rifampicin. Overall, in this much larger dataset we were not able to identify further resistance determinants associated with homoplasic nucleotide positions outside candidate genes.

Figure 5 suggests one approach to integrating the algorithm into a routine laboratory workflow. Here, phenotypic predictions based on whole-genome sequencing would be made for isolates containing resistance determinants, only benign mutations, or no relevant mutations. As data accrue, confidence in the characterisation of each mutation will grow to the point where routine phenotyping can be restricted to isolates containing uncharacterised mutations that prevent phenotypic prediction. Phenotyping is likely to persist longer for some drugs than for others: although katG S315T and rpoB S450L were the dominant mutations for isoniazid and rifampicin, we identified many infrequently occurring resistance-determining mutations in $p n c A$, as also shown in another study. ${ }^{30}$

Limitations to this study include the few isolates that are phenotypically resistant to second-line drugs. Nevertheless, we show that the algorithm can be successfully applied across the full range of drugs studied, and that the characterisation of mutations as benign is as important as the characterisation of resistance determinants. A further limitation is that we did not have the resources to systematically rephenotype and resequence discordant isolates. However, the size of the study does allow the penetrance of mutations to be assessed across many isolates, mitigating the effects of phenotypic error. Moreover, because the presence of the high-level resistance determinant katG S315T in isoniazid-susceptible isolates has been proposed as a marker of sample mislabelling, our rate of nine (1.9\%) of 480 discordant isolates compares favourably with previous reports. ${ }^{2}$ Nevertheless, this limitation increases the difficulty of assessing the importance of drug-resistance determinants outside the 23 candidate genes versus phenotypic error or mislabelling to our false-susceptible predictions. Finally, the training and validation sets were drawn from different populations as a consequence of availability at different times. However, our simulation study showed the robustness of our approach.

Public Health England has started to do whole-genome sequencing in parallel to workflows to assess its suitability as a one-stop diagnostic platform for mycobacterial infections. Parallel phenotypic drugsusceptibility testing will lend support to the status of some mutations, and characterise further ones. The cosmopolitan nature of tuberculosis in the UK will enhance our understanding of molecular determinants

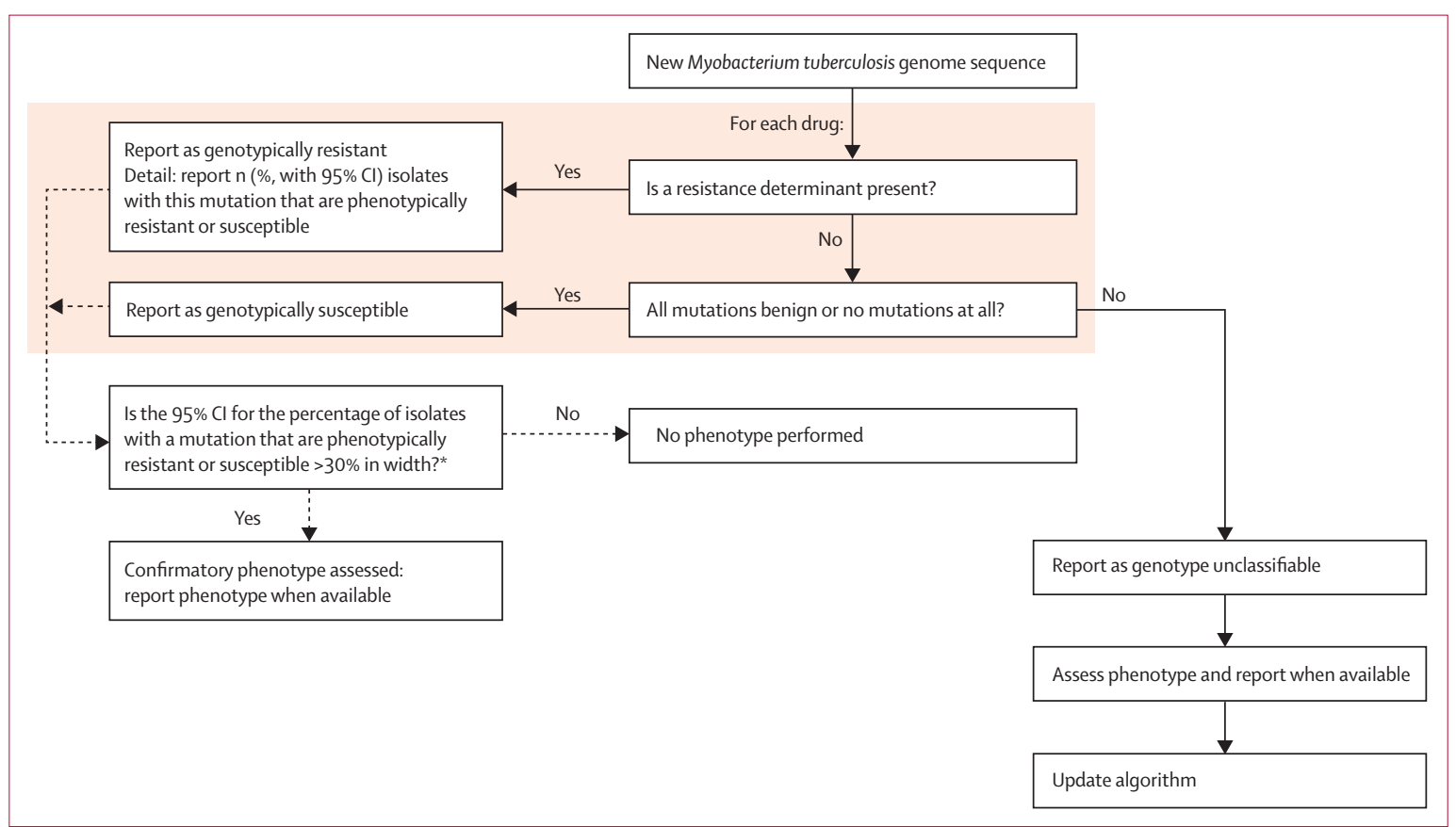

Figure 5: Proposed workflow for transition towards whole-genome sequencing-based drug-susceptibility testing

*The $30 \% \mathrm{Cl}$ width suggested is arbitrary, and represents how the precise proportion of isolates with a mutation is probably less relevant than understanding whether this proportion is very high, moderate, or low. However, the precise width could be determined by what is regarded as an acceptable degree of clinical risk, and could also vary by the estimate of proportion resistant. For example, with a targeting width of less than $30 \%$, ten phenotypically resistant isolates of ten isolates with a mutation (100\%) has a lower $97.5 \% \mathrm{Cl}$ of $69 \%$, so mutations that are uniformly resistant would need to be phenotyped 11 times before confirmatory phenotyping would stop. For a mutation associated with resistance in $50 \%$ of isolates, phenotyping would need to happen 48 times, and for a mutation associated with resistance in either $25 \%$ or $75 \%$ isolates, 36 times. 
of resistance, as will the global accumulation of data from whole-genome sequencing. Rollout of the GeneXpert diagnostic test for $M$ tuberculosis DNA and resistance to rifampicin has set a precedent for the deployment of advanced technology in low-income, highburden settings; ${ }^{31}$ with cost-effective, field-ready, sequencing platforms such as the Oxford Nanopore MinION on the horizon, ${ }^{32}$ the prospect of delivering drug-susceptibility testing based on whole-genome sequencing globally, including to settings where no phenotypic drug-susceptibility testing currently exists, is a possibility. Outbreaks could be detected from the same data at no additional cost, potentially adding to local tuberculosis control. ${ }^{11}$ Advances in software now enable sequence data analysis and interpretation to take place without the need for skills in bioinformatics, ${ }^{33,34}$ removing this obstacle to rollout. As techniques to extract genomic DNA for whole-genome sequencing from primary samples improve, ${ }^{35}$ the main remaining scientific challenge to the success of drug-susceptibility testing based on whole-genome sequencing will therefore be the composition of a comprehensive catalogue of characterised mutations.

\section{Contributors}

TMW, TAK, SVO, JH, CDOE, DJW, GK, CLCI, RB, FAD, PS, DWC, EGS, ASW, NI, SN, and TEAP designed the study. TMW, TAK, SVO, SF, GK, FAD, RD, DWC, EGS, NI, and SN obtained the samples. TMW, TAK, SVO, GK, CA-B, CG, JP, PS, NI, and SN did the DNA extraction and sequencing. TAK, SVO, SF, FAD, EGS, NI, and SN did the phenotyping. TAK, SVO, SF, GK, RD, EGS, NI, and SN obtained the metadata. TMW, TAK, SVO, JH, CDOE, PB, ZI, SF, KEN, DJW, DAC, GK, CLCI, RB, FAD, PS, DWC, EGS, ASW, NI, SN, and TEAP analysed the data. TMW, TAK, SVO, JH, CDOE, PB, ZI, SF, KEN, DJW, DAC, GK, CLCI, RB, FAD, CA-B, CG, JP, RD, PS, DWC, EGS, ASW, NI, SN TEAP drafted the manuscript. The MMM Informatics Group (JD, CC, MA, LM-M, DE, DW, MM, and TG) provided the informatics and computational foundations from which this study could be done.

\section{The MMM Informatics Group}

Jim Davies, Charles Crichton, Milind Acharya, Laura Madrid-Marquez, David Eyre, David Wyllie, Tanya Golubchik (all University of Oxford, Oxford, UK), and Melinda Munang (University of Warwick, Coventry, UK).

\section{Declaration of interests}

PS is a consultant for Genoscreen. CG is, and CA-B was, an employee of Genoscreen. JP has received support for conference travel and accommodation from Illumina. DAC is funded by a research fellowship from the Royal Academy of Engineering and Balliol College, Oxford. TMW is an MRC Research Training Fellow. PB has a Genomic Medicine and Statistics Wellcome Trust DPhil studentship. ZI and DJW are Wellcome Trust and Royal Society Sir Henry Dale Fellows. KEN is a Rhodes Scholar, Rhodes Trust and part of the RCUK Digital Economy Programme grant number EP/G03861/1 (centre for Doctoral Training in Healthcare Innovation). DWC and TEAP are NIHR senior investigators. All other authors declare no competing interests.

\section{Acknowledgments}

This study was funded by the UK Clinical Research Collaboration (Wellcome Trust [grant 087646/Z/08/Z], Medical Research Council, National Institute for Health Research [NIHR grant G0800778]); NIHR Oxford Biomedical Research Centre, NIHR Oxford Health Protection Research Unit on Healthcare Associated Infection and Antimicrobial Resistance (grant HPRU-2012-10041), Health Innovation Challenge Fund (UK Department of Health and the Wellcome Trust [grant T5-358]), Wellcome Trust Sanger Institute core funding (grant 098051), EU FP7 Patho-Ngen-Trace (grant FP7-278864-2). We thank the High-Throughput Genomics Group at the Wellcome Trust Centre for Human Genetics (funded by Wellcome Trust grant $090532 / \mathrm{Z} / 09 / \mathrm{Z}$ ) for the generation of the sequencing data. All sequences are available in NCBI or ENA (or both), phenotypes and archive accession numbers are in the supplementary tables (appendix 2). This report is independent research by the NIHR. The views expressed in this publication are those of the authors and not necessarily those of the UK National Health Service, the NIHR, or the Department of Health.

References

1 WHO. Global tuberculosis report 2014. http://apps.who.int/iris/ bitstream/10665/137094/1/9789241564809_eng.pdf?ua=1 (accessed Nov 28, 2014).

2 Casali N, Nikolayevskyy V, Balabanova Y, et al. Evolution and transmission of drug-resistant tuberculosis in a Russian population. Nat Genet 2014; 46: 279-86.

3 Feng Y, Liu S, Wang Q, et al. Rapid diagnosis of drug resistance to fluoroquinolones, amikacin, capreomycin, kanamycin, and ethambutol using genotype MTBDRsl assay: a meta-analysis. PLoS One 2013; 8: e55292.

4 Drobniewski F, Nikolayevskyy V, Maxeiner H, et al. Rapid diagnostics of tuberculosis and drug resistance in the industrialized world: clinical and public health benefits and barriers to implementation. BMC Med 2013; 11: 190.

5 Daum LT, Rodriguez JD, Worthy SA, et al. Next-generation ion torrent sequencing of drug resistance mutations in Mycobacterium tuberculosis strains. J Clin Microbiol 2012; 50: 3831-37.

6 Köser CU, Bryant JM, Becq J, et al. Whole-genome sequencing for rapid susceptibility testing of $M$ tuberculosis. N Engl J Med 2013; 369: 290-92.

7 Clark TG, Mallard K, Coll F, Preston M, Assefa S. Elucidating emergence and transmission of multidrug-resistant tuberculosis in treatment-experienced patients by whole genome sequencing. PLoS One 2013; 8: e83012.

8 Farhat MR, Shapiro BJ, Kieser KJ, et al. Genomic analysis identifies targets of convergent positive selection in drug-resistant Mycobacterium tuberculosis. Nat Genet 2013; 45: 1183-89.

9 Comas I, Coscolla M, Luo T, et al. Out-of-Africa migration and Neolithic coexpansion of Mycobacterium tuberculosis with modern humans. Nat Genet 2013; 45: 1176-82.

10 Walker TM, Lalor MK, Broda A, et al. Assessment of Mycobacterium tuberculosis transmission in Oxfordshire, UK, 2007-12, with whole pathogen genome sequences: an observational study. Lancet Respir Med 2014; 2: 285-92.

11 Walker TM, Ip CL, Harrell RH, et al. Whole-genome sequencing to delineate Mycobacterium tuberculosis outbreaks: a retrospective observational study. Lancet Infect Dis 2013; 13: 137-46.

12 Lunter G, Goodson M. Stampy: a statistical algorithm for sensitive and fast mapping of Illumina sequence reads. Genome Res 2011; 21: 936-39.

13 Li H, Handsaker B, Wysoker A, et al. The sequence alignment/map format and SAMtools. Bioinformatics 2009; 25: 2078-79.

14 Iqbal Z, Caccamo M, Turner I, Flicek P, McVean G. De novo assembly and genotyping of variants using colored de Bruijn graphs. Nat Genet 2012; 44: 226-32.

15 Stamatakis A. RAxML version 8: A tool for phylogenetic analysis and post-analysis of large phylogenies. Bioinformatics 2014; 30: 1312-13.

16 Didelot X, Wilson DJ. ClonalFrameML: efficient inference of recombination in whole bacterial genomes. PLoS Comput Biol 2015; 11: e1004041.

17 Chewapreecha C, Marttinen P, Croucher NJ, et al. Comprehensive identification of single nucleotide polymorphisms associated with beta-lactam resistance within pneumococcal mosaic genes. PLoS Genet 2014; 10: e1004547.

18 Sreevatsan S, Pan X, Zhang Y, Kreiswirth BN, Musser JM. Mutations associated with pyrazinamide resistance in pncA of Mycobacterium tuberculosis complex organisms. Antimicrob Agents Chemother 1997; 41: 636-40.

19 Cole ST, Brosch R, Parkhill J, et al. Deciphering the biology of Mycobacterium tuberculosis from the complete genome sequence. Nature 1998; 393: 537-44.

20 Zhang Z, Wang Y, Pang Y, Kam KM. Ethambutol resistance as determined by broth dilution method correlates better than sequencing results with embB mutations in multidrug-resistant Mycobacterium tuberculosis isolates. J Clin Microbiol 2014; 52: 638-41.
See Online for appendix 2 
21 Brossier F, Veziris N, Jarlier V, Sougakoff W. Performance of MTBDR plus for detecting high/low levels of Mycobacterium tuberculosis resistance to isoniazid. Int J Tuberc Lung Dis 2009; 13: 260-65.

22 Brossier F, Veziris N, Aubry A, Jarlier V, Sougakoff W. Detection by GenoType MTBDRsl test of complex mechanisms of resistance to second-line drugs and ethambutol in multidrug-resistant Mycobacterium tuberculosis complex isolates. J Clin Microbiol 2010; 48: 1683-89.

23 Gillespie SH, Crook AM, McHugh TD, et al. Four-month moxifloxacin-based regimens for drug-sensitive tuberculosis. N Engl J Med 2014; 371: 1577-87.

24 Merle CS, Fielding K, Sow OB, et al. A four-month gatifloxacincontaining regimen for treating tuberculosis. N Engl J Med 2014; 371: 1588-98.

25 Böttger EC. The ins and outs of Mycobacterium tuberculosis drug susceptibility testing. Clin Microbiol Infect 2011; 17: 1128-34.

26 Angeby K, Juréen P, Kahlmeter G, Hoffner SE, Schön T. Challenging a dogma: antimicrobial susceptibility testing breakpoints for Mycobacterium tuberculosis. Bull World Health Organ 2012; 90: 693-98

27 Loman NJ, Misra RV, Dallman TJ, et al. Performance comparison of benchtop high-throughput sequencing platforms. Nat Biotechnol 2012; 30: 434-39.

28 Koller D, Friedman N. Probabilistic graphical models. http://mitpress.mit.edu/books/probabilistic-graphical-models (accessed Nov 11, 2014).
29 Petrella S, Gelus-Ziental N, Maudry A, Laurans C, Boudjelloul R, Sougakoff W. Crystal structure of the pyrazinamidase of Mycobacterium tuberculosis: insights into natural and acquired resistance to pyrazinamide. PLoS One; 6: e15785.

30 Miotto P, Cabibbe AM, Feuerriegel S, et al. Mycobacterium tuberculosis pyrazinamide resistance determinants: a multicenter study. MBio 2014; 5: e01819-14.

31 Weyer K, Mirzayev F, Migliori GB, et al. Rapid molecular TB diagnosis: evidence, policy making and global implementation of Xpert MTB/RIF. Eur Respir J 2013; 42: 252-71.

32 Eisenstein M. Oxford Nanopore announcement sets sequencing sector abuzz. Nat Biotechnol 2012; 30: 295-96.

33 Steiner A, Stucki D, Coscolla M, Borrell S, Gagneux S. KvarQ targeted and direct variant calling from fastq reads of bacterial genomes. BMC Genomics 2014; 15: 881.

34 Bradley P, Gordon NC, Walker TM, et al. Rapid antibiotic resistance predictions from genome sequence data for $S$ aureus and M tuberculosis. CSH Lab Journals, 2015. http://dx.doi. org/10.1101/018564.

35 Doughty EL, Sergeant MJ, Adetifa I, Antonio M, Pallen MJ. Culture-independent detection and characterisation of Mycobacterium tuberculosis and $M$ africanum in sputum samples using shotgun metagenomics on a benchtop sequencer. PeerJ 2014; 2: e585. 